\title{
Note on Collision Frequency of Snowflakes
}

\author{
By M. Fujiwara \\ Meteorological Research Institute, Tokyo, Japan \\ (Manuscript received 31 May 1957)
}

\begin{abstract}
Collision frequency of snow aggregates is discussed from some simple estimations. Estimation shows that, if cohesion condition exists, a rapid growth by aggregation may occur, throughout crystal and aggregation stages.
\end{abstract}

\section{Introduction}

In the previous paper [1] the present author has described some evidence which indicates the importance of collision between snowflakes in the mechanism working in the development of size-distribution of precipitation elements. Namely, the following observed phenomena have led us to the expectation of a frequent collision, (1) simultaneous changes in the numbers of large and small flakes usually are observed on the ground, (2) giant aggregates are produced by some coherence process within a thin melting layer.

Shapes and structures of individual snowflakes are not simple. Variation in aggregate form will result in that of the fall velocity. Some of snowflakes, certainly, fall fluttering or rotating. This complexity in motion may cause both the relative velocity and the collision cross-section and consequently the frequency of collision to increase appreciably. The former problem is discussed later in section 3. But, if there exist no such an effect that a snowflake dodge about another, the influence of random motion upon the mean collision cross-section may be insignificant. So the orientations and accordingly also the cross-sections of flakes under the study will be assumed to remain constant through the sweeping.
So far, values of collision frequency as to raindrops have been estimated by some investigators. Therefore, evaluation of the ratio of collision frequency of snowflakes to. that of raindrops will be made.

\section{Fall velocities of snowflakes}

Fall velocities of snow crystals are given by Nakaya [2] first, and as to snow aggregates by Magono [3], Langleben [4], Imai, Fujiwara, Ichimura and Toyama [5]. Magono's data in his report are expressed in terms of apparent size, while Imai et al's and Langleben's in terms of melted diameter. Recently, Imai [6] has made a discussion concerning the fall velocity of snow aggregates by replotting Magono's data for melteddiameter. In these results individual velocities seem to be extremely scattered around. the mean curves which are provided by experimental formulas. The present author tried to give a theoretical basis for the observed velocity, and some reasonable expressions of parameters in $v-D$ formulas. were derived.

If the shape of a particle remains constant. through its fall, the drag coefficient $C_{d}$ depends only on Reynolds number of the particle. It is expressed by $C_{d}(d \cdot v)$ under constant air density $\sigma$ and viscocity, where $d$ and $v$ are the apparent diameter and the velocity of the particle, respectively. As 
the drag force varies proportionally to the cross section and the square of velocity of the particle, the equation which determines the fall velocity becomes

$$
(\sigma / 2) .(\pi / 4) d^{2} \cdot v^{2} C_{d}(d \cdot v)=m g
$$

where $m$ and $g$ are the mass of the particle ard the acceleration of gravity, respectively. Concerning $C_{d}$, an assumption was made that it can be substituted by a power of Reynolds number, $a d^{n} v^{n}$, where $a$ and $n$ are constants. Then, equation (1) becomes

$$
v=\left(\frac{4 g}{3 a \sigma}\right)^{1 /(n+2)} \frac{D^{3 /(n+2)}}{d},
$$

where $D$ is the melted diameter of the particle. If the shape of the particle is assumed to be spheroidal, its volume can be expressed by $(\pi / 6) p d^{3}$ where $p$ is the flatness of the spheroid. Using this assumption (2) is rewritten as

$$
v=\left(\frac{4 g}{3 a \sigma}\right)^{1 /(n+2)} p^{1 / 3} \cdot \rho^{1 / 3} \cdot D^{(1-n) /(n+2)},
$$

or

$$
\begin{aligned}
& v=K D^{1 / s}, \text { where } K=(4 g / 3 a \sigma)^{1 /(n+2)} \cdot p^{1 / 3} \cdot \rho^{1 / 3}, \\
& s=(n+2) /(1-n) .
\end{aligned}
$$

Spilhaus and Langleben have given empirical formulas of fall velocity for rain drops and snowflakes, respectively, and further Imai $e t$ al have got, from their measurements, a formula similar to that of Langleben for snow. It is found that the form of equation $\left(3^{\prime}\right)$ includes the results obtained by the above authors. That is, $s$ will take a value of about 2 for raindrops after Spilhaus [7] and about 3 for snowflakes after Langleben or Imai et al. From equation (3) or $\left(3^{\prime}\right)$, it is also found that the fall velocity varies proportionally to the cubic root of the density of the particle, if the particles have a geometrical similarity. From the above equation, $n$ becorres 0.0 and 0.25 for raindrops and snowflakes, respectively. Obviously, this relation agrees, for raindrops, with the experimental results on the drag coefficient of sphere given by many investigators, that is, the coefficient is almost constant in the range of Reynolds number between akout 400 and 3000 , while for srowflakes the drag coefficient varies approximately as

$$
C_{d}=\text { const. }(d \cdot v)^{1 / 4}
$$

in about the same range of Reynolds num. ber.

Since the function form of $C_{d}(d v)$ obviously depends only on the shape of the particle under constant air density, $n=0$ will also apply approximately to some sphere-like particles as graupel or spatial dendrites and $n=0.25$ to ordinary aggregate flakes. ${ }^{*}$ Using the representative values of two experimental results for $K$ after Spilhaus, Langleben and Imai et al, that is, 207 for snowflakes and 140 for raindrops, we are led to the expression for $K$ as follows:

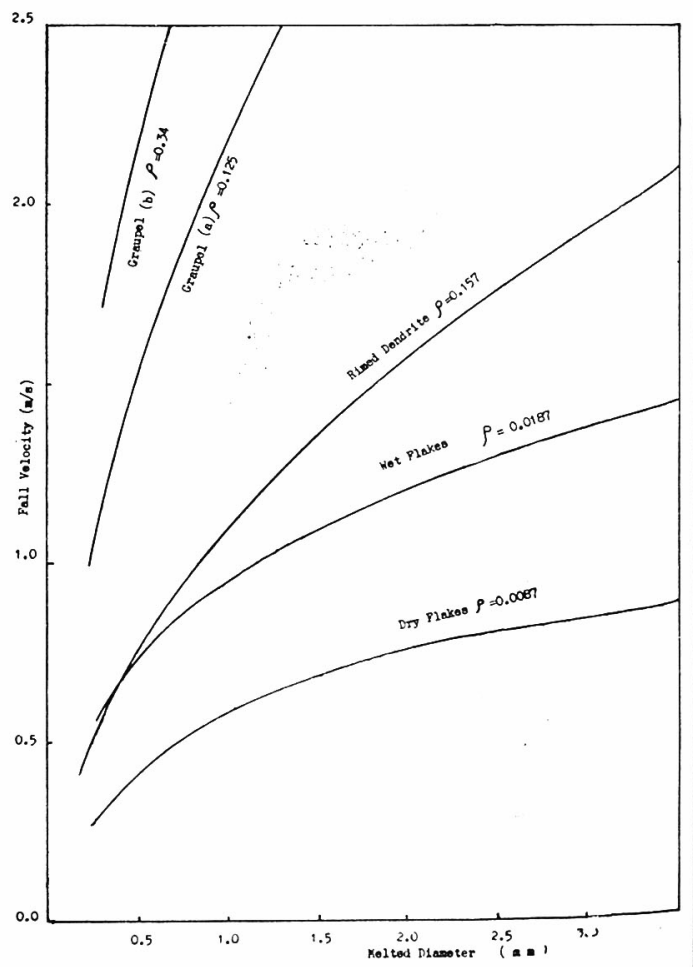

Fig. 1 Curves of fall velocity of snow aggregates versus melted diameters calculated from equation $v$ $=K D^{1 / s}$. Values of parameters are shown in Tabl 1 .

$$
K_{\text {obl.sph. }}=778 \rho^{0.333}
$$

and $\quad K_{\text {sphere }}=140 \rho 0.333$

where the unit of velocities and diameters are in C. G.S. and for snowflakes $\rho=0.0187$

* $n$ is variable according to the shape, exceeding 0.25 for more plate-like and less than this for more spheroidal aggregate. $(a$

$\mathrm{F}$

$\mathrm{ca}$

al

$\mathrm{Vt}$

$\mathrm{sn}$

$\mathrm{F}$

he

th

of

at

th

$\mathrm{dr}$

for

$\mathrm{m}$

at

th

be

is,

th

for

$\mathrm{COI}$

$\mathrm{Th}$

by

for

lea

to

pro

als

pa

$\mathrm{mi}$

ag

Sug 
Table 1. Physical properties of aggregate forms used in calculations.

num.

iously

article

1 also

re-like

es and

Using

rimen-

gleben

flakes

to the

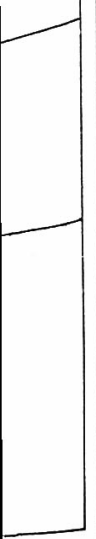

gregates

bation 0

Tabl 1

(4)

meters

$=0.0187$

xceeding this for

\begin{tabular}{|c|c|c|c|c|c|c|}
\hline Aggregate forms & $\rho\left(\mathrm{g} / \mathrm{cm}^{3}\right)$ & Authority & Shape & $\begin{array}{l}\text { Diameter } \\
\text { factor* } \\
\mathrm{k}\end{array}$ & $\stackrel{\mathrm{K}}{\text { (C.G.S.) }}$ & $\mathrm{s}$ \\
\hline Dry Flake & 0.0087 & C. Magono[3] & oblate & 6.65 & 160 & 3 \\
\hline Wet Flake & 0.0187 & $" 1$ & $" 1$ & 3. 70 & 207 & 3 \\
\hline Rimed Dendrite & 0.0157 & $" 1$ & sphere & 3.61 & 351 & 2 \\
\hline Graupel (1) & 0.125 & U. Nakaya [2] & $" 1$ & 2.0 & 700 & 2 \\
\hline$(2)$ & 0.34 & I. Imai[8] & $" 1$ & 1.43 & 977 & 2 \\
\hline
\end{tabular}

* Defined in Section 3.

(after Magono's wet flakes) is applied. From these equations various values of $K$ can be calculated, and the typical ones are shown in Table 1. The resultant fall velocities, thus obtained for some typical snow aggregates, are also illustrated in Fig. 1.

Table 1 also contains the values of $\rho$ used here and their original authors.

Langleben found from his observed data that at temperatures below $32 F$ the value of $K$ for dendrite aggregates was 160 while at $37 F$ it was 207 . These agree well with the present results given in Table 1 (for dry flakes).

Langleben has given also an experimental formula, $v=366 D^{0.611}$, for snow-aggregate mixture of plates and dendrites, and has attributed the rapid increasing teature of the curve to the difference in observed numbers between small dendrites and plates. It is, however, shown from the value of $K$ in the third row of the table that if the aggregate form is spatial the velocity curve will become similar to that obtained by Langleben. Therefore, in order to obtain the $v$ - $D$ curves by experiments, observation of the aggregate form is very important in every respect.

Magono has considered some effect of air leakage through a snowflake which will lead to decrease its drag coefficient. This may probably act in light dendrite flakes and also in single crystals. Though for these particles the values of $K$ introduced here might, therefore, be reduced somewhat, the agreem.ent with Langleben's formulas may suggest this effect to be insignificant. Since exact quantitative data for this problem have not yet been published, more detailed discussions will be placed aside here.

Some of the Magoro's $v-d$ curves [3] for horizontally oriented flakes become flat at about 2-cm of apparent diarreter and then gradually decrease with further ircrease of diameter, though the measurements are widely scattered around the curve. For such large flakes the plain type would have resulted due to successive coalescences at the edges, under the condition with temperature $0.8 \mathrm{C}$ which is surely favorable to coherence. Therefore, the curve seems to show a transition from the spheroidal stage given by equation (3) to the stage of plain type, in which $v$ rather decreases with size as can be seen from equation (11).

The above theoretical treatment, therefore, though simplified, will provide reliable results in approximation.

\section{Ratio of collision factors of snow to that of rain}

In fact, fall velocities of snow are scatter ed widely around the mean curve as mentioned above, which will cause collision even snow particles of equal sizes. In the previous paper [1] the ratio of the collison factor of snow to that of rain was given for various aggregation forms. This has been calculated from mean $v-D$ curves. Of course, if the large variance in velocity arises, the total collision frequency will be affected greatly by this and become larger than that given in the previous paper. The contribution rate by the random component 
obviously depends on the variance rather than the slope of mean curves if the latter is small. The previous results, therefore, may give some minimum, except for spherical type in which some over-estimation of frequency would have been made due to its improper velocities. The effect of the variarce on the collision factor will be discussed quantitatively elsewhere.

Now, collision frequency of snowflakes per unit distance of its fall is given by the following equation.

$$
p_{D D^{\prime}}=F_{D} F_{D^{\prime}} S_{D D^{\prime}} \Delta v_{D D^{\prime}} / \bar{v}_{D D^{\prime}},
$$

where suffix $D$ or $D^{\prime}$ indicates the particles having melted diameters $D$ or $D^{\prime}$ (hereafter expressed by $D$ or $D^{\prime}$-particles), $p_{D D^{\prime}}$, the collision frequency between $D$ and $D^{\prime}$ particles per unit volume, $F_{D}$ or $F_{D^{\prime}}$, spatial concentration of particles per unit diameter interval, $S_{D D^{\prime}}$, the sweeping crosssection of $D^{\prime}$ by $D$-particles and $\Delta v_{D D^{\prime}}$ the relative fall velocity. ${ }^{\dagger}$ Considering a stationary precipitation through layers containing snow ard rain where the precipitation rate remains constant, say, $(\pi / 6)$ $D^{3} V_{D} F_{D}=$ const., the calculations were made. Although there are evidences that size distribution of precipitation particles in melting layer may change, these details were omitted for the approximation, and $V_{D} F_{D}=$ const. is assumed.

The cross-section of snowflakes $S^{*}{ }_{D D^{\prime}}$ is expressed by the melted diameters as

$$
S^{*}{ }_{D D^{\prime}}=(\pi / 4) \cdot\left(D+D^{\prime}\right)^{2} \cdot k^{2}
$$

where $k$ is the ratio of apparent size of snow aggregate to the melted diameter. This diameter factor, $k$, can be calculated from the shape, density and orientation of falling

\footnotetext{
$\dagger$ In order to get true collision frequency, it is necessary to calculate $p_{D D^{\prime}} E_{D D^{\prime}}$, where $E_{D D^{\prime}}$ is collision efficiency between $D$ and $D^{\prime}$-particles, similar to that given by Langmuir for drops. The data for snow collision efficiency have not yet been obtained at all because of its complexity. Evaluation, here, is confined only to $p_{D D^{\prime}}$ disregarding $E$, and the following discussion will be made.
}

particle. In some types, a disk with thickness $1 / 5$ its diameter and in others sphere was assumed. As to the mean density the values given in the author's contributions listed in Table 1 were used. Horizontal orientations were assumed in all cases. Now, the diameter factor obtained in each aggregate form is shown in Table 1 . The fall velocities were calculated from equation (3) and (4) using $K$ and $S$ shown in the Table. The quantity $\Delta v / \bar{v}$ in $(5)$ is given, approximately, by logarithmic finite differential of $v(D)$, then $(1 / 2) \cdot(\Delta D / \bar{D})$ and $(1 / 3) \cdot(\Delta D / \bar{D})$ are obtained for sphere and disk respectively.

From equation (5), ratios of collision fator of snow to rain in unit fall depth become as follows according to the aggregate form,

$$
p_{D D^{\prime}}{ }^{*} / p_{D D^{\prime}}=\frac{2}{3}\left(v_{D} v_{D^{\prime}} / v_{D}{ }^{*} v_{D^{\prime}}{ }^{*}\right) \cdot k^{2}
$$

for disk-like snow aggregate and

$$
p_{D D^{\prime}}{ }^{*} / p_{D D^{\prime}}=\left(v_{D} v_{D^{\prime}} / v_{D}{ }^{*} v_{D^{\prime}}{ }^{*}\right) \cdot k^{2}
$$

for spherical aggregate, where * is attached to the quantities for srow. Substituting for $v_{D} v_{D^{\prime}}$, the square of $v_{D}$, these ratios were calculated, which are shown in Table 3 .

Table 3. Ratio of collision frequency of snow aggregates to raindrops, $P_{D D^{\prime}} * / P_{D D^{\prime}}$

\begin{tabular}{l|ccc}
\hline & Melted & Diameter & $(\mathrm{mm})$ \\
& 1.0 & 2.0 & 3.0 \\
\hline Dry Flake & 1018 & 1286 & 1470 \\
Wet Flake & 183 & 243 & 278 \\
Rimed Dendrite & & 206 & \\
Graupel (a) & \multicolumn{3}{|c}{} \\
(b) & \multicolumn{3}{|c}{4.2} \\
\hline
\end{tabular}

At a glance of the results in the table, largeness of collision factor in snowy stage is evident. $\dagger$ Especially, in the dry flakes,

+ Some discrepancies of the individual values in the table from those given in the previous investigation [1] are seen. This is due to the improvement in application of fall velocity, that is, in previous calculation a single $v-D$ curve was applied throughout as representative, while in the present, individual $v-D$ curves were introduced for respective aggregation forms. It is considered, however, that these discrepancies do not change the essential part of the discussion made here. extre with

4. $\mathbf{E}$ o]

Th aggre raind are si 10001 of $\mathrm{fa}$ : layer factor latter the 1 was $n$ Gunn with 
extremely frequent collisions are expected, with the collision ratio of the order of $10^{3}$.

\section{Effect of collision on size distribution of snowflakes}

The result in Table 3 shows rapid snowaggregate growth results in comparison with raindrop if cohesion conditions on the particle are satisfied. For dry flakes with the facter 1000 the ircollision frequency in only $10 \mathrm{~m}$ depth of fall corresponds to that of raindrops in a layer $10000 \mathrm{~m}$ thick. For wet flakes with the factor $250,40 \mathrm{~m}$ snow layer corresponds to the latter. For simplicity a rough estimation of the modification of raindrop size distribution was made based on the curves given by Rigby, Gunn and Hitschfeld [9] (Fig. 5 of the paper), with the result as shown in Table 4 .

Table 4. Modification of raindrop distribution by coalescence alone through $2000 \mathrm{~m}$ fall in the rate of $4.5 \mathrm{~mm} / \mathrm{h}$ (A)

\begin{tabular}{l|ccc}
\hline Diameter (mm) & 1 & 2 & 3 \\
\hline (A) $\log _{10}\left(\mathrm{~N}-\mathrm{N}^{\prime}\right)$ & +0.21 & -0.16 & -0.22 \\
\hline (B) $\left.\log _{10}\left(\mathrm{~N}-\mathrm{N}^{\prime}\right)\right|_{50^{*}}$ & +0.13 & -0.95 & -1.38 \\
\hline $\begin{array}{l}\text { (N is the concentration of particle in unit } \\
\text { diameter interval and } N^{\prime} \text { is the modified } \\
\text { concentration.) }\end{array}$
\end{tabular}

The third row of Table 4 shows the corresponding modification to the second row for wet flakes for $50 \mathrm{~m}$ fall, under the condition the all collisions result in coalescence. This result is reached on the tentative assumption of constant modification rate, though true process may be revealed by successive or differential calculus for fall length.

Correct expression of collision frequency involves collision efficiency $E$. This has been estimated near 1 for rain by Langmuir and others. As to snow, in spite of its much importance, estimation of $E$ are entirely lacking. This quantity, therefore, has not so far been placed in discussion. The results in the table, however, suggest that, even if the frequency of coalescence or disintegration is by one order lower than that of collision, this would be obviously very sig- nificant for size-distributions of precipitation elements. There are also evidences that giant snow aggregates fall beneath the $\mathrm{OC}$ level, this probobly resulted within the thin layer through coalescences.

The effects of the turbulent motion in the free atmosphere are not inquired here.

Generally, an increase in the factor is expected with the increase of the total sweeping length. But if dodging habit were accompanied with turbulent flow, these two effects caused by turbulence might act to cancel each other to some extents.

\section{Fall velocities of snow crystals}

Snow crystals will be classified into three major-types, i. e., spatial, plane and line. Snow crystals such as small ice particles, powder snow and spatial dendrites fall under the first type, which may be treated as sphere. The plane type will include single plane dendrites, plates and stars while the line type needles and columns. For the treatment of the velocity equation in analogy to Section 2, the assumption of horizontal orientation of the falling particles was also made. When the crystal is of plane type, its growth is considered to be confined, generally, in the plane directions, that is, the relation between the apparent diameter and the diameter of equivalent spherical drop will become as follows:

$$
d^{-1}=(3 t \rho / 2)^{1 / 2} \cdot D^{-(3 / 2)}
$$

where $t$ is the thickness of the crystal and other notations are the same as in Section 2. Substituting (9) in (2), the following equation results,

$$
v=K \cdot D^{1 / s}
$$

where $K=(4 \mathrm{~g} / 3 \mathrm{a} \sigma)^{1 /(n+2)} \cdot(3 t / 2)^{1 / 2} \cdot \rho 1 / 2$

and $s=-3 n / 2(n+2)$

In this relation, if the drag coefficient remains as in snowflake, substituting $n=0.25$ in this equation, the following formula is obtained.

$$
v=\text { const } \cdot D^{-0.167} \cdot \rho^{1 / 2} \text {. }
$$

This shows that the fall velocity decreases with the increase of size. Since the dimen- 
sion of snow crystal is usually far smaller than that of snow aggregate applied in determination of $n$, equation (11) will not always be correct for snow crystals. In Nakaya's diagram on the fall velocity of snow crystal, single dendrites have a constant speed. This means that $n$ becomes zero in equation (10). Therefore, it is suggested from the data and the above equation that the drag coefficient $C_{d}(d v)$ for the plate is kept nearly constant down to the region of lower Reynolds numbers such as scores.

In needles, under the similar assumptions of horizontal orientations and only one $\mathrm{di}-$ mensional growth, the following equation results by means of the analogical treatment to $(10)$,

$$
v=\text { const } \cdot D^{-3 n /(n+2)}
$$

Nakaya has found that the fall velocity of needles increases rapidly with the mass. Drag coefficient $C_{d}$ of small needle is not found, but, from the data (i.e. Smithsomian Physical Tables 1933 P. 201) concerning sphere which cover a wide range of Reynolds number, $n$ is expected to be a negative fraction of unity in the transition region from aerodynamical to viscous flow. If it is so, from equation (12), the data given by Nakaya on needles seem to agree with the theory.

\section{Cross-section and collision frequency of snow crystals}

The assumption of horizontal orientation was also applied to snow crystals. In this case, an avoiding effect of collision would be less probable than in aggregates because partial air stream through the particles may occur. The collision cross-section of a dendri. tic single-crystal is obviously almost equal to that of enveloping circle(Fig. 1(A)). But, in the case of line type as needles, a question arises, as to how the equivalent cross-section becomes.

The mean collision probability between ideal needles was evaluated and represented in terms of the equivalent cross-section. Let two ideal needles have lengths of $2 b$ and $2 a \mathrm{~cm}$ (where $b>a$ ) as indicated in Fig. 4. When $b \gg a$, the probability approaches to that in the well-known Buffon's problem. where $b$ is infinitely large whereas $a$ is finite In the present problem the lengths of two needles are of the comparative order. In Fig 4. (c), the angle $\theta$ in which collision takes place, is clearly a function of length $a, b$, the distance between the centers of the needles tion of analysis in Fig. longer
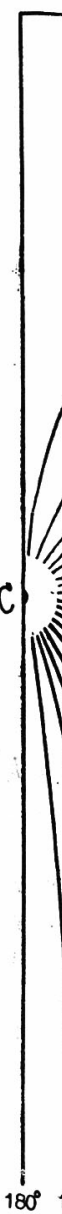

$180^{\circ}$ A

Fig 3 which larger ponds to section $\mathrm{i}$ $\theta / \pi$ wi the resu section In dend section, by the Nakay lations crystal 
needles $d$, and the direction $\phi$. The calculation of $\theta(a, b, \phi)$ was made by elementary analysis and then the result was illustrated in Fig. 3, where $C$ indicates an end of the longer needle and the distance $A B$ corres-

$\mathrm{n}$ this

would ecause $S$ may lendri: equal But, restion section etween sented tion. $2 b$ and ig. 4. hes to oblem, finite. of two r. In ollision length of the

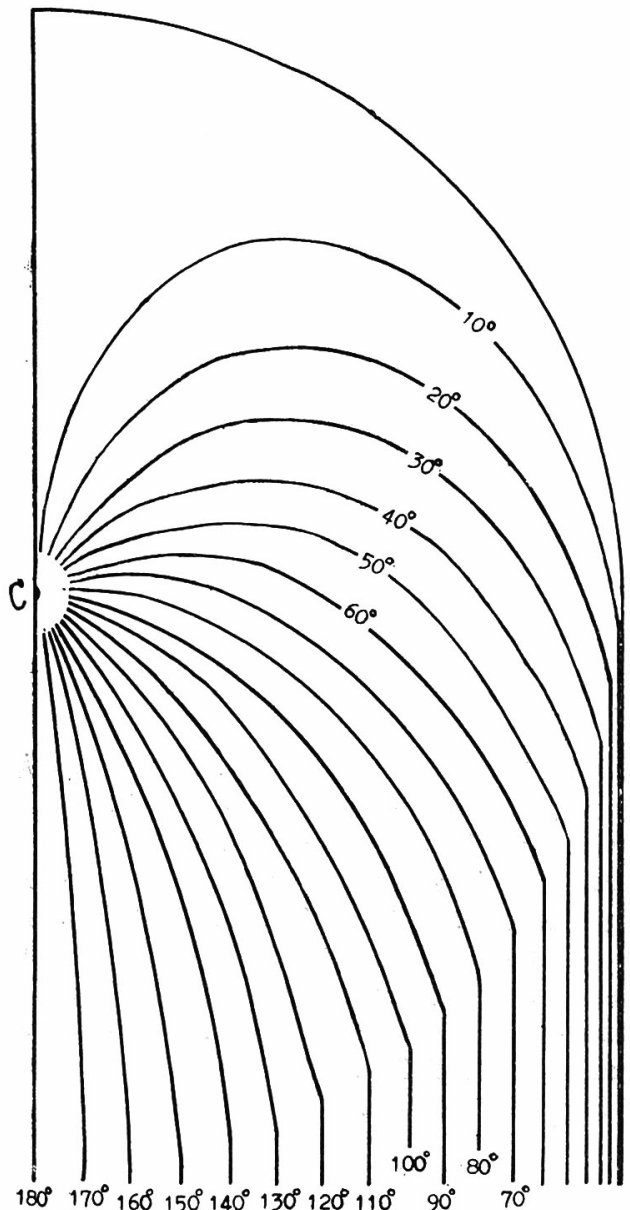

A

Fig 3 Calculated values of angle $\theta$ within which collision must occur around the end of larger needle, $C$.

ponds to the length $a$. The probability crosssection is obtained by integrating the amount $\theta / \pi$ with respect to area. Table 5 shows the results, which give the probability crosssection of needles in $\mathrm{cm}^{2}$.

In dendrites, the equivalent collision crosssection, as mentioned above, is substituted by the envelope.

Nakaya originally has given empirical relations between mass and dimension for some crystal forms [2] based on his observations
Table 5. Probability cross-section of needle $(\mathrm{cm})^{2}$

\begin{tabular}{c|cccc}
\hline$r$ & \multicolumn{4}{|c}{$a(\mathrm{~cm})$} \\
& 0.01 & 0.05 & 0.1 & 0.3 \\
\hline 0.1 & 0.00244 & 0.0610 & 0.244 & - \\
0.3 & 0.00082 & 0.0205 & 0.082 & 0.74 \\
0.5 & 0.00050 & 0.0125 & 0.050 & 0.45 \\
0.7 & 0.00036 & 0.0090 & 0.036 & 0.32 \\
1.0 & 0.00025 & 0.0063 & 0.025 & 0.23 \\
\hline
\end{tabular}

in Hokkaido, Japan, namely, $m=0.0038 d^{2}$ (mgr) in plane dendrites and $m=0.0029 d$ (mgr) in needles, where $d$ is the apparent diameter or length in $\mathrm{mm}$. Applying the relations, comparison of the collision crosssections was made between the three crystal forms. The ratios of the cross-section of plane dendrite and needle to that of equivalent spherical particle are shown in Table 6 , where each cross-section taken was of a pair of equal masses and forms.

From the table, each snow crystal is found to have a cross-section amounting to more than several tens of times that of spherical drops in some range of diameter. For the needles, a rapid increase of it is seen between 200 and 300 microns in size $D$ due to the increase in their length. When $D=$ 400 and 300 microns, $d$ becomes about 3 and $5 \mathrm{~mm}$ respectively. Up to such sizes aggregates are usually observed in Japan. Thus, the collision cross-section must be a significant factor in these size ranges. If the conditions for the coagulation of snow crystals through collision and the actual distribution of their velocities were found, the real rates of growth would be easily evaluated. Even if the exact process of mutual binding of crystals is still unknown, these extreme cross-section ratio would be of importance for precipitation generation. The present author has reported in the previous paper [1], from the evidences of radar observation, that moderate precipitations were found to occur from relatively low level with a temperature of about $-5 C$, where the condition would be favorable to the development of needles. 
Accordingly, these results concerning the cross-section would give us a new problem of precipitation growth in the generating cells.

\section{Acknowledgements}

The author expresses his hearty thanks to Dr. I. Imai, Prof. K. Isono and Mr. K. Suda for their kind guidance and in structive advices, and also to Mrs. T. Yanase and Miss. T. Inoue for their assistances.

Table 6 Comparison of collision cross-sections of typical crystal forms

\begin{tabular}{cccc}
\hline $\begin{array}{c}D \\
\text { Dia. }\end{array}$ of Sphere & Mass of Particle & $\begin{array}{c}m \\
\text { Ratio of Cross Section } \\
\text { of Dendrite to Sphere }\end{array}$ & $\begin{array}{c}S_{\text {Seedl. }} / S_{s p h .} \\
\text { Ratio of Cross Section } \\
\text { of Needle to Sphere }\end{array}$ \\
\hline 10 microns & .000523 micro-grams & 1.38 & - \\
50 & .0654 & 6.89 & - \\
100 & .523 & 13.77 & 2.59 \\
150 & 1.177 & 20.65 & 13.9 \\
200 & 4.188 & 27.6 & 40.8 \\
300 & 14.13 & 41.2 & 239. \\
400 & 33.5 & 55.1 & $(665)$ \\
500 & 65.4 & 68.9 & - \\
1000 & 523.0 & $(137.7)$ & - \\
\hline
\end{tabular}

\section{References}

1) Fujiwara, M. 1957: Freliminary investigation of snowflake size-distribution Pap. Meteor. Geophys. 9. (to be published). -2) Nakaya, U. 1949: Study of snow. Iwanami Shoten, Tokyo. (in Japanese), - 3) Magono, C. 1953: Falling velocity of snowflakes. Research on Snow \& Ice, I. (in Japanese). -4) Imai, I.,M. Fujiwara, I. Ichimura and Y. Toyama. 1955: Radar reflectivity of falling snow. Pap. Meteor. Geophys. 6. 130. -5) Langleben, M. P. 1954: The terminal velocity of snow aggregates. McGill Univ., Sci. Rep. MW-16. -6) Imai, I. 1956: Radar and snowfall. (unpublished). -7) Spilhaus, A. F. 1948: Drop size, intensity and radar echoes of rain. J. Meteor. 5, 161 164. -8) Imai, I. 1950: A study of the monsoon snowtall at Wajima. J. Meteor. Soc. Japan, II, 33, 9. -9) Rigby, E. C., K. S. L. Gunn, and W., Hitshfeled, 1954: Development during fall of rain-drop size Distributions. McGill Univ. Sci. Rep. MW-17, Jan. 